\title{
Dynamics of Ischemia-Dependence as Neurodegeneration
}

\author{
Lawrence MA* \\ Department Of Pathology, University of Malta Medical School, Mater Dei Hospital, \\ Europe
}

*Corresponding author: Lawrence M Agius, Department Of Pathology, University Of Malta Medical School, Mater Dei Hospital, Tal-Qroqq, Msida, 27 “Ballarat”, Guzeppe

\author{
Review Article
Volume 2 Issue 4
Received Date: July 10, 2018
Published Date: August 07,2018
DoI: $10.23880 /$ mjccs- 16000166
}

Caruana Street, Tal-Virtu, Rabat, Rbt09, Malta, Europe, Tel: 356-21451752; Email: lawrence.agius@um.edu.mt

\begin{abstract}
Proportional dimensions of molecular misfolding and unfolding afford a profile understanding of a basic neuronal degeneration that is also reflected in the endoplasmic reticulum (ER) response to aberrant degradation of various molecular profiles. The distributional attributes for further progression of proportional attributes arise in terms of a protein aggregation phenomenon that assigns the neurofibrillary dimensions of injury to an ischemic network of neurons in a manner that accounts for specific manifestations of neuronal apoptosis. Incremental profiles for further recharacterization of performance dynamics are well-indexed by systems biology of transfer between endoplasmic reticulum and the Golgi apparatus. In realized evolutionary course for such neurodegeneration, the attributes for further progression to accelerated course further promote permissive dimensions as well-characterized by a failure of homeostatic control for further change in molecular transfer and distribution.
\end{abstract}

Keywords: Neurodegeneration; Misfolding; Aggregation; Protein; Ischemia

\section{Abbreviations: ER: Endoplasmic Reticulum.}

\section{Introduction}

The unfolded endoplasmic reticulum response (UPR) implicates dynamics of protein degradation that specifically promotes cell apoptosis in response to ischemia-induced degradation-defective mechanisms of onset and progression in gradual neuronal cell death. It is in such terms that ubiquitination of proteins such as alpha-synuclein dynamically promotes inclusion body formation and accumulation in neurons in Parkinson's disease. C. elegant modeling includes loss of dopamine neurons, disrupted dopamine-dependent behavior, increased sensitivity to stress, age-dependent aggregation and movement deficits as in Parkinson's disease [1]. In Huntington's disease, the appearance of clinical signs, inclusion formation, and neurodegeneration progress independently [2].

The nuclear presence and aggregation of expanded ataxin-3 are critical in pathogenesis of spinocerebellar ataxia type 3 with linkage to the nuclear pore machinery [3]. Neuroinflammation and the presence of amyloid beta protein and neurofibrillary tangles are critical lesions in Alzheimer's disease [4]. Mathematical models describe 


\section{Medical Journal of Clinical Trials \& Case Studies}

both microscopic processes during misfolded protein aggregation and large-scale events indicating progression of neurodegeneration misfolded protein-mediated disease [5]. There is an association of aneuploidy with Alzheimer's pathology in the hippocampus and anterior cingulate cortex and neuronal degeneration in the substantial nigra [6]. Each isoform of metallothioneins has a protective effect against disease and affects intractable disease such as amyotrophic lateral sclerosis and spinal muscular atrophy [7].

\section{Linkage Dynamics}

Linkage dynamics in both neurons and glia allow for the evolutionary history of protein aggregation to form inclusion bodies in terms beyond the system profiles of the Endoplasmic Reticulum luminal accumulation of unfolded and misfolded protein molecules. The molecular basis of protein misfolding and aggregation is central to a full comprehension in the pathogenesis of neurodegeneration and for the development of therapeutical strategies [8]. In such conceptual dynamics, derivation of inclusion bodies in several forms of ischemia-dependent neurodegeneration emerge as profiles that go beyond simple apoptotic response. The aggregation of neurodegeneration-related disease proteins in cerebral schema indicates molecular overlap between neurodegeneration and ischemic stroke [9]. p27Kip1 regulates transcription by associating its $\mathrm{COOH}$ region to the transcriptional regulators on the chromatin and by the NH2-domain to cyclin-cdk complexes; it then phosphorylates the specific targets on the chromatin leading to gene expression [10]. Provocation of the indeterminate accumulation of multiple protein molecular species characterize essential attributes of a neurodegenerative cascade in terms of accumulated biophysical dimensions for further driven protein unfolding and misfolding. Aggregation of fused in sarcoma (FUS) protein and mutated FUS gene induce neurodegeneration that includes amyotrophic lateral sclerosis and frontotemporal dementia [11]. Nicotinamide function includes the general maintenance of cellular energy levels and the more specific inhibition of nicotinamide adenine dinucleotide-dependent deacetylase sirtuin 1 [12].

\section{Endoplasmic Reticulum Evolution}

Strictly operative increments in ER evolution arise primarily in the constitutive response to proteins that overwhelm the ubiquitin proteosome system that in turn further augments unfolding and misfolding of protein molecules. Store operated $\mathrm{Ca}^{2+}$ entry may promote cytosolic $\mathrm{Ca}^{2+}$ oscillations which in turn support cell survival [13].

Protein phase transition is a novel way of how neurons compartmentalise and regulate biochemical reactions and may be useful in cell cycle regulation, membrane-less organelle biogenesis and protein aggregation in neurodegenerative states [14]. In a real sense, the dynamics for apoptotic cell death includes neurofibrillary tangle formation in terms of onset/progression of aberrant phosphorylation of tau molecules in Alzheimer disease and as a synucleinopathy in Parkinson's disease. Octadecylpropyl sulfatide, a PPARalpha agonist, reduces neurodegeneration and restores the memory deficits caused by hypoxia-ischemia in mice [15]. Also, the evolution of prion diseases allows for protease dysfunction as a specific determinant in inclusion body accumulation in a manner that is dynamically reflective especially of protein aggregation. Unique misfolded conformations of tau, referred to as strains, are hypothesised to underlie distinct neuroanatomic and cellular distribution of pathologic tau aggregates [16].

The various aspects concerning prolongation of polyglutamine traits formulate emergent profiles that in unique terms are permissive for manifestations of a lesion that outlines the further incipient dynamics towards definition of the aggregation-linked phenomena for incremental accumulation of specific species of misfolding events. The etiologic link between metabolic alteration and the observed diseases such as neurodegeneration and cancer is still elusive [17]. It is within the system profiles of folding events that protease activity is induced by aberrant response to such molecular moieties of betaamyloid and alpha-synuclein, or as expression of endoplasmic reticulum attributes of biophysical accumulation. Phospholipid alterations in the brain are related to progressive neurodegeneration and cognitive impairment after injury [18].

Polyglutamine insert expansion is closely allied to the dimensions for further misfolding as indeed reflected in anticipation and progression in disease severity in Huntington's disease and further promulgated by systems biology of misfolding of various specific molecular species as terms of reference to such polyglutamine tract expansion. Molecular chaperones, such as the small heat shock proteins, are key elements of the proteostasis 


\section{Medical Journal of Clinical Trials \& Case Studies}

network with inhibition of aggregation of misfolded proteins [19].

\section{Inclusion Body Definition}

Inclusion body definition of neurodegeneration permits the expression for an accumulative phenomenon in its own right in a manner that profiles substantial attributes as neuronal cell death. p27Kip1 modulates alpha-synuclein expression [20]. The performance attributes of protein molecular aggregation arise as systemically prolonged failure of the ubiquitinproteosomal dimensions of an apoptotic response that outlines specifically the proportional processes of molecular expansion as reflected in both causative genes and protein expanded traits.

Biophysics of molecular accumulation within neurons and/or glia is a promotional attribute for neuronal dysfunction that is directly linked to the specific terms of characterization of the endoplasmic reticulum luminal accumulation within the established constitution of the ubiquitin proteosomal response.

These are best re-characterized as dimensions of profiled resistance to failed or aberrant ER degradation. It is thus within the promotional events for protein molecular accumulation that the specific formulas for recharacterization permit ischemic dimensions of a neurodegenerative series of cascade events in neuronal cell death and apoptosis. Alzheimer's disease is the commonest tauopathy and involves progressive accumulation of amyloid-beta and hyperphosphorylated tau; bigenic models parallel the pathological course of AD in models of transgenic mice over-expressing APP and Triple Repeat Tau [21].

\section{Performance Dynamics}

Performance dynamics of a generalized neuronal cell response is apparently the distributional pattern recognition of chaperone dysfunction in further determined dynamics that include the phosphorylation of tau as neurofibrillary degeneration. It is profile index suppression of protein molecular accumulation that defines best the neurodegeneration of neurons in terms of significant re-distribution of systems of ubiquitination and as incremental accumulation of the dimensional attributes for further accumulation of aggregated protein moieties within cells. Fibrous cross-beta aggregates (amyloids) and their transmissible forms (prions) induce disease and control heritable traits in yeast [22].

Hence, in real measure, the performance dimensions projected as cellular system response is integral to the profile outline and proportionality of molecular expansion that incrementally account for the specific emergence of aggregated molecules of re-characterized ischemic dimensions. The full spectrum of molecular factors that promote AD pathogenesis is not known. White matter abnormalities, and in particular myelin and oligodendrocytes, could be mechanistically important in AD pathology and could be potential treatment targets [23]. However non-coding RNAs have been implicated in both the onset and pathogenesis of AD [24].

The simple formulated increment of molecular aggregation is a highly permissive accumulation that includes also defining terms of aggregation as wellprojected in terms of misfolded or unfolded molecules such as alpha synuclein or polyglutamine traits. Expansion of polyglutamine increases the propensity for Huntingtin protein aggregation, a process known to be implicated in neurodegeneration [25].

\section{Deterministic Profiles}

Performance homeostasis is hence integral to a generic neurodegeneration that is constitutively deterministic profile for substantial attribution gain or loss as specifically outlined by a biophysical accumulation of prion particles. It is thus defined in terms that permit constitutional re-characterizaiton of the ischemiadependent neuronal apoptotic response. Failed performance dimensions of neurons and glia are candidate profiles of a neurodegenerative cascade attributable to an apoptosis that is systemic dimension for the misfolding/unfolding evolution in further recharacterization of molecular events. The distributional attributes for aberrant aggregation are therefore symptomatic of a release dimension of molecular specificity. Monitoring endomembrane integrity indicates rescued entry of tau seeds into the cytosol and thereby prevents seeded aggregation [26].

\section{Neuronal Loss}

Pertinent to the overall ischemia-dependent loss of neurons that creates characterized neuronal groups is a process that links directly the aggregation of ER luminal dimensions that extends to the evolutionary traits of the 


\section{Medical Journal of Clinical Trials \& Case Studies}

Golgi apparatus. The distinctive dimensional profile of neurodegenerative traits is thus integral to the promotional attempts at molecular characterization that promotes the system expansion of molecules and the promotional events in performance dynamics of the degenerating neurons. Factors contributing to pathologic tau phosphorylation are not well understood but protein phosphatase $2 \mathrm{~A}$ is dysregulated in tauopathies of progressive supranuclear palsy and Alzheimer disease [27]. It is the dimensional disproportion of the prion particle as an aggregated molecule that best permits dimensions for the performance attributes of an aberrant ischemia/aggregation event. It is apparently within the specific profile of performance dynamics that neurons interact with glia in an overall profile re-characterization in prion disease. Determined identification of neurodegeneration expose the neuronal networks and also the individual neurons as system responses in recharacterization of performance dynamics as wellprofiled by the ER response to a dysfunctional ubiquitinproteosomal system. This in turn induces variant performance dynamics of aberrant molecular accumulation and aggregation. Beta-synuclein changes in Lewy body disease may exacerbate neuronal dysfunction caused by accumulation of alpha-synuclein by affecting protein degradation pathways [28].

\section{Concluding Remarks}

Performance indices of progression of the neurodegeneration are best exemplified as failed systems of response in terms of neuronal homeostasis. Some reports show that microglia are implicated in neurodegeneration and oxidative stress generation, whilst others indicate that microglia do not respond to ischemia/reperfusion injury [29]. Reduced RNA binding protein TIA1 protects against tau-mediated neurodegeneration in vivo [30]. More recent evidence suggests acetylation of tau protein at lysine 280 may be a critical step in molecular pathology in Alzheimer's disease, Pick's disease, frontotemporal dementia and progressive supranuclear palsy [31]. As such, the incremential incorporation of permissive events includes the dimensional spread of neuronal cell death that is proportional to the accumulation of aggregated or expanded traits of molecular species. The emergence for the evolutionary scope for such protein aggregation is a specific profile for the performance attributes of an injury that primarily includes system distribution of aggregated misfolded proteins. Such misfolding is reflected also in unfolded protein species that critically encompass dimensions for molecular ischemic characterization and re-characterization of the given neurodegenerative state.

\section{References}

1. Cooper JF, Van Raemsdonk JM (2018) Modeling Parkinson's disease in C. elegans. J Parkinsons Dis 8(1): 17-32.

2. Kielar C, Jennifer Morton A (2018) Early neurodegeneration in R6/2 mice carrying the Huntington's disease mutation with a superexpanded CAG repeat, despite normal lifespan. J Huntingtons Dis 7(1): 61-76.

3. Sowa AS, Martin E, Martins IM, Schmidt J, Depping R, et al. (2018) Karyopherin alpha-3 is a key protein in the pathogenesis of spinocerebellar taxia type 3 controlling the nuclear localisation of ataxin-3. Proc Natl Acad Sci USA 115(11): E2624-E2633.

4. Maiti P, Paladugu L, Dunbar GL (2018) Solid lipid curcumin particles provide greater anti-amyloid, antiinflammatory and neuroprotective effects than curcumin in the $5 \mathrm{xFAD}$ mouse model of Alzheimer's disease. BMC Neurosci 19(1): 7.

5. Carbonell F, Iturria-Medina Y, Evans AC (2018) Mathematical modeling of protein Misfolding mechanisms in neurological diseases: a historical overview. Front Neural 9: 37.

6. Shepherd CE, Yang Y, Halliday GM (2018) Region- and cell-specific aneuploidy in brain raging and neurodegeneration. Neuroscience 374: 326-334.

7. Nakamura S, Shimazawa M, Hara H (2018) Physiological roles of metallothioneins in central nervous system diseases. Biol Pharm Bull 41(7): 1006-1013.

8. Villar-Pique A, Schmitz M, Candelise N, Ventura S, Llorens F, et al. (2018) Molecular and clinical aspects of protein aggregation assays in neurodegenerative diseases. Mol Neurobiol.

9. Kahl A, Bianco I, Jackman K, Baskar J, Milaganur Mohan H, et al. (2018) Cerebral ischemia induces the aggregation of proteins linked to neurodegenerative diseases. Sci Rep 8(1): 2701.

10. Bachs O, Gallastegui E, Orlando S, Bigas A, MoranteRedelat JM, et al. (2018) Role of p27Kip1 as a 


\section{Medical Journal of Clinical Trials \& Case Studies}

transcriptional regulator. Oncotarget 9(40): 262592627.

11. Matsumoto T, Matsukawa K, Watanabe N, Kishino Y, Kunugi H, et al. (2018) Self-assembly of FUS through its low-complexity domain contributes to neurodgeneration. Hum Mol Genet 27(8): 1353-1365.

12. Fricker RA, Green EL, Jenkins SI, Griffin SM (2018) The influence of nicotinamide on health and disease in the central nervous system. Int J Tryptophan Res 11: 117864918776658.

13. Lang F, Petzi L, Hauser S, Hermann A, Stournaras C, et al. (2018) To die or not to die SGK1-sentive ORAI/STIM in cell survival. Cell Calcium 74: 29-34.

14. Boeynaems S, Tompa P, Van Den Bosch L (2018) Phasing in on the cell cycle. Cell Div 13: 1.

15. Kossatz E, Silva-Pofia D, Suarez J, de Fonseca FR, Maldonado R, et al. (2018) Octadecylpropyl sulfamide reduces neurodegeneration and restores the memory deficits induced by hypoxia-ischemia in mice. Front Pharmacy 9: 376.

16. Gibbons GS, Banks RA, Kim B, Changolkar L, Riddle DM, et al. (2018) Detection of Alzheimer disease (AD)-specific tau pathology in $\mathrm{AD}$ and NonAD tauopathies by immunohistochemistry with novel conformation-selective tau antibodies. J Neuropathol Exp Neural 77(3): 216-228.

17. Sade D, Shaham-Niv S, Arnon ZA, Tavassoly O, Gazit E (2018) Seeding of proteins into amyloid structures by metabolic assemblies may clarify certain unexplained epidemiological associations. Open Biol 8(1).

18. Sabogal-Guaqueta AM, Posada-Duque R, Cortes NC, Arias-Londofio JD, Cardona-Gomez GP (2018) Changes in the hippocampal and peripheral phospholipid profiles are associated with neurodegeneration hallmarks in a long-term global cerebral schema model: attenuation by Linalool. Neuropharmacology 135: 556-571.

19. Cox D, Whiten DR, Brown J, Horrocks MH, San Gil R, et al. (2018) The small heat shock protein Hsp27 binds alpha-synuclein fibrils, preventing elongation and cytotoxicity. J Biol Chem 293(12): 4486-4497.
20. Gallastegui E, Domuro C, Serratosa J, Darrieux A, Sin L, et al. (2018) p27Kip1 regulates alpha-synuclein expression. Oncotarget 9(23): 16368-16379.

21. Arner A, Rockenstein E, Mante M, Florio J, Masliah D, et al. (2018) Increased vulnerability of the hippocampus in transgenic mice over expressing APP and triple repeat tau. J Alzheimers Dis 61(3): 12011219.

22. Chandramowlishwaran P, Sun M, Casey KL, Romanyuk AV, Grizel AV, et al. (2018) Mammalian amyloidogenic proteins promote prion nucleation in yeast. J Biol Chem 293(9): 3436-3450.

23. Nasrabady SE, Rizvi B, Goldman JE, Brickman AM (2018) White matter changes in Alzheimer's disease: a focus on myelin and oligodendrocytes. Acta Neuropathol Commun 6(1): 22.

24. Idea ML, Munk R, Abdelmohsen K, Gorospe M (2018) Noncoding RNAs in Alzheimer's disease. Wiley interdiscip Rev RNA 9(2).

25. Kim DK, Cho KW, Ahn WJ, Perez-Acufia D, Jeong H, et al. (2017) Cell-to-cell transmission of polyglutamine aggregates in C. elegans. Exp Neurobiol 26(6): 321328.

26. Falcon B, Noad J, McMahon H, Random F, Goedert M (2018) Galectin-8-mediated selective autophagy protects against seeded tau aggregation. J Biol Chem 293(7): 2438-2451.

27. Park HJ, Lee KW, Oh S, Yan R, Zhang J, et al. (2018) Protein phosphatase $2 \mathrm{~A}$ and its methylation modulating enzymes LCMT- 1 and PME- 1 are dysregulated in tauopathies of progressive suprnuclear plays and Alzheimer disease. J Neuropathol Exp Neural 77(2): 139-148.

28. Evans T, Kok WL, Cowan K, Hefford M, Anichtchik O (2018) Accumulation of beta-synuclein in cortical neutrons is associated with autophagy attenuation in the brains of dementia with Lewy body patients. Brain Res 1681: 1-13.

29. Surinkaew P, Sawaddinuk P, Apajjal N, Chattipakom N, Chattipakom SC (2018) Role of microglia under cardiac and cerebral ischemia/reperfusion (I/R) injury. Metab Brain Dis 33(4): 1019-1030. 


\section{Medical Journal of Clinical Trials \& Case Studies}

30. Apicco DJ, Ash PEA, Maziuk B, LeBang C, Medalla M, et al. (2018) Reducing the RNA binding protein TIA1 protects against tau-mediated neurodegeneration in vivo. Nat Neurosci 21(1): 72-80.
31. Lucks-Wold B, Seidel K, Udo R, Omalu B, Ornstein M, et al. (2017) Role of tau acetylation in Alzheimer's disease and chronic traumatic encephalopathy: the way forward for successful treatment. J Neurol Neurosurg 4(2). 